\title{
Low-Grade Oligodendroglioma of the Pineal Region: Case Report
}

\author{
Fabricio Correa Lamis ${ }^{1, *}$ Manoel Antonio de Paiva Neto ${ }^{1, *}$ João Norberto Stavale ${ }^{2}$ Sergio Cavalheiro \\ ${ }^{1}$ Department of Neurosurgery, Federal University of São Paulo, São \\ Paulo, Brazil \\ 2 Department of Pathology, Federal University of São Paulo, São \\ Address for correspondence Manoel Antonio de Paiva Neto, MD, \\ Department of Neurosurgery, Federal University of São Paulo, São
} Paulo, Brazil,

Both authors contributed equally to this work

J Neurol Surg Rep 2015;76:e55-e58.

\begin{abstract}
Keywords

- oligodendroglioma

- pineal region

- tectal plate

- pineal gland

Background Although germ cell tumors and pineal cell tumors account for most of the histologic tumor subtypes, $>17$ different tumors can arise in this location. We report a rare case of a low-grade oligodendroglioma that arose in the pineal region.

Clinical Presentation A young woman complaining of a headache underwent magnetic resonance imaging that showed a mass in the pineal region and mild hydrocephalus. A ventriculoperitoneal shunt was performed followed by a near-total tumor removal, due to tumor invasion of the tectal plate and thalamus. The histologic examination confirmed the diagnosis of a low-grade oligodendroglioma. The patient then underwent chemotherapy and radiotherapy as adjuvant therapies.

Conclusion Although the pineal region is a common place for a large number of tumoral lesions, low-grade oligodendrogliomas are extremely rare in this location. This case is only the second account of a benign oligodendroglioma of the pineal region reported in the literature.
\end{abstract}

\section{Introduction}

Pineal region tumors correspond to 3 to $8 \%$ of brain tumors in children and $<1 \%$ in adults. ${ }^{1-3}$ Although germ cell tumors and pineal cell tumors account for the great majority of histologic tumor subtypes, $>17$ different tumors can arise in this location. We report a rare case of low-grade oligodendroglioma (OD) in the pineal region treated by almost total surgical resection, followed by chemotherapy and radiotherapy with complete remission. This is the fifth case of OD and the second case of grade II OD in the pineal region reported in the literature ( $\mathbf{- T a b l e ~} \mathbf{1})$. $^{1,4,5}$

\section{Case Report}

A 22-year-old woman was admitted to the hospital with a 1-month history of progressive headache. Her past medical history revealed a congenital deafness secondary to rubella. Her neurologic examination showed bilateral deafness, upward gaze palsy, mydriasis, convergence impairment, and no pupil light reaction (complete Parinaud syndrome). She underwent brain magnetic resonance imaging (MRI) that disclosed a heterogeneous, irregularly enhancing solid lesion in the region of the pineal gland, with compression of the cerebral aqueduct ( - Fig. 1). Mild ventriculomegaly was also noted. Imaging of the remainder neuroaxis revealed no other lesions, and the search for alfa-fetoprotein and $\beta$-human chorionic gonadotropin in the cerebrospinal fluid and blood was negative.

The patient underwent a ventricular-peritoneal shunt on an urgent basis, but her visual symptoms did not improve. Afterward, she was submitted to a supracerebellar-infratentorial approach to the pineal tumor in the semisitting position. This approach was chosen due to the main anteroposterior lesion received

May 13, 2014 accepted after revision

October 19, 2014

published online

February 5, 2015
DOI http://dx.doi.org/

10.1055/s-0034-1396653. ISSN 2193-6366.
Copyright @ 2015 by Georg Thieme

Verlag KG, Inc., Stuttgart - New York Tel: +1(212) 584-4662.
License terms

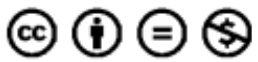


Table 1 Cases of oligodendroglioma of the pineal region

\begin{tabular}{|l|l|l|l|l|}
\hline Study & Age, y/Sex & Histology & Symptoms & Treatment \\
\hline${\text { Levidou et } \mathrm{al}^{5}}^{5}$ & $37 / \mathrm{F}$ & Low-grade OD grade II & Gaze impairment & Total resection \\
\hline Das et $\mathrm{al}^{4}$ & $59 / \mathrm{F}$ & Anaplastic OD & $\begin{array}{l}\text { Confusion, headache, memory } \\
\text { disturbance }\end{array}$ & $\begin{array}{l}\text { Total resection, RT, } \\
\text { and CH }\end{array}$ \\
\hline Kumar et al $^{17}$ & $30 / \mathrm{M}$ & Anaplastic OD & NA & NA \\
\hline Kumar et al & (7) & Anaplastic OD & NA & NA \\
\hline Current study & $22 / \mathrm{F}$ & Low-grade OD grade II & Gaze impairment, headache & $\begin{array}{l}\text { Subtotal resection, } \\
\text { RT, and CH }\end{array}$ \\
\hline
\end{tabular}

Abbreviations: $\mathrm{CH}$, chemotherapy; NA, not available; OD, oligodendroglioma; RT, radiotherapy.

axis, and there was no significant projection laterally or in the direction of the cerebellum mesencephalic fissure.

After the core of the tumor was debulked, the tumor was separated from the veins of Galen and Rosenthal. The tumor presented a well-defined cleavage plan in its anterior projection into the third ventricle, but showed an infiltrative pattern to the tectorial plate and pulvinar of the thalamus bilaterally. Tumor removal was conducted up to the superior colliculi in the tectal plate, where a small tumor remnant was left behind to avoid brainstem invasion, and no clear cleavage plane was recognized.

A microscopic examination of hematoxylin-and-eosin stained sections showed round neoplastic cells with characteristic perinuclear haloes and centrally located round nuclei with open chromatin and without any pleomorphism or necrosis (-Fig. 2). An immunohistochemical analysis showed that the neoplastic cells were positive for synaptophysin, neurofilaments, and glial fibrillary acidic protein. The Ki-67 proliferation index was low (1\% of neoplastic cells). The fluorescence in situ hybridization analysis (chromosome

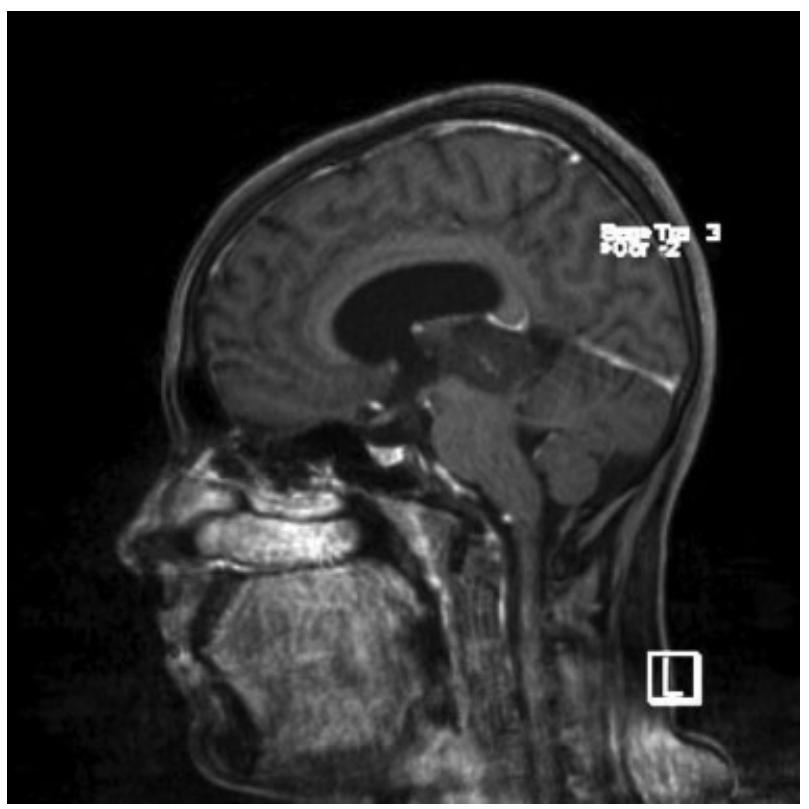

Fig. 1 Preoperative midline sagittal postgadolinium magnetic resonance imaging presented a heterogeneous solid lesion in the pineal region extending to the posterior part of the third ventricle. arms $1 p$ and $19 q)$ showed $1 p$ and $19 q$ deletion. Therefore, a diagnosis of OD, grade II, was assigned.

The patient had an uneventful postoperative course but persisted with upward gaze palsy, although her pupillary light reflex improved. The postoperative MRI showed a small residual tumor in the tectorial region (-Fig. 3). The patient was discharged home on postoperative day 5 . Because of the incomplete resection and the infiltrative tumor pattern observed during the surgical procedure, the patient underwent fractionated external-beam radiation therapy to the tumor bed (50.4 Gy over 28 fractions), with a concurrent course of temozolomide(TMZ) $\left(75 \mathrm{mg} / \mathrm{m}^{2} /\right.$ day) followed by five cycles of TMZ(150 mg/m²/day for 5 days every 28 days) as an adjuvant therapy. A MRI obtained at her 24-month followup demonstrated no evidence of disease. The patient remained with an upward gaze palsy (-Fig. 4).

\section{Discussion}

The pineal region is limited dorsally by the splenium of the corpus callosus and tela choroid, ventrally by the quadrigeminal plate and mesencephalus, rostrally by the posterior third ventricle, and caudally by the cerebellar vermis. ${ }^{3}$ Pineal-region tumors can arise from the neoplastic transformation of cells presented in structures in the area, such as pineal parenchyma,

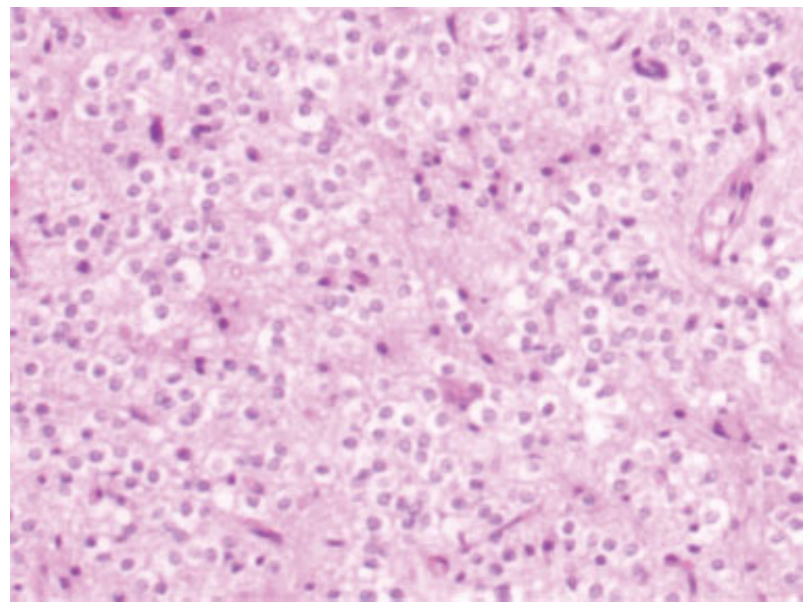

Fig. 2 Microscopic sections of tumor showing round neoplastic cells with perinuclear haloes and a centrally located round nucleus with open chromatin. 


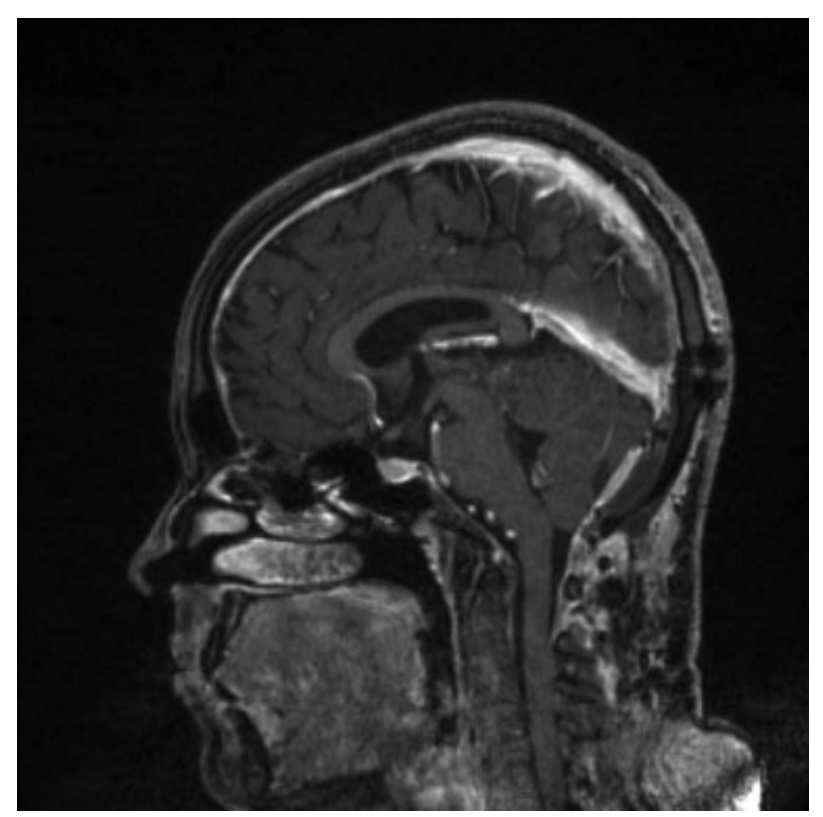

Fig. 3 Early postoperative midline sagittal postcontrast magnetic resonance imaging showing a small residual tumor in the tectal plate region.

supportive pineal, glial, arachnoid, ependymal, or thalamic cells. ${ }^{6}$ Only 11 to $28 \%$ of pineal-region tumors arise from the pineal parenchyma itself. Germinative tumors, which represent 50 to $75 \%$ of the lesions in this region, originate from primitive germ cell rests that are retained in the pineal region. Other miscellaneous and less frequent histologic types can be found in this small area including lymphoma, metastasis, craniopharyngiomas, and lipomas, among others. Although oligodendrocytes can be found in the pineal gland tissue and in the adjacent brainstem, ${ }^{5}$ the cellular origin of OD is not well defined. There are two theories on the cellular origin of ODs:

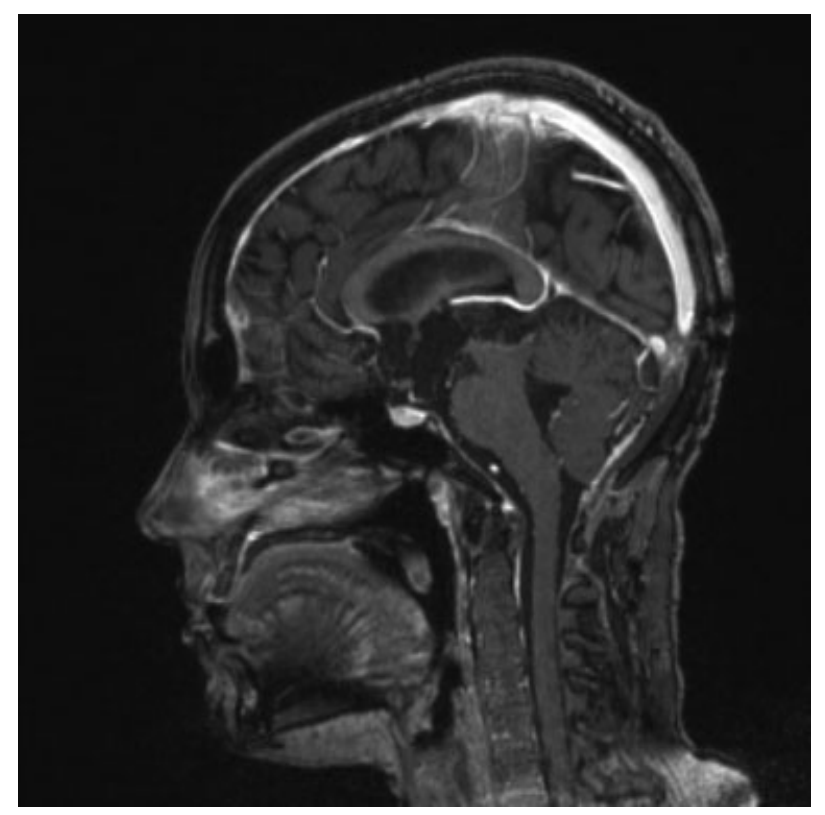

Fig. 4 Two-year postoperative sagittal postcontrast magnetic resonance imaging after radiotherapy and chemotherapy demonstrated no visible residual tumor.
They can arise from either dedifferentiated mature cells or glial progenitor cells, also known as a cancer stem cell that has been found in gliomas. ${ }^{7}$

ODs constitute 5 to $20 \%$ of all glial tumors. They are predominantly a tumor of adulthood, with a peak incidence between the fourth and sixth decades of life. ${ }^{8-11}$ Most ODs arise in the white matter of the cerebral hemispheres, predominantly in the frontal lobes. They diffusely infiltrate brain tissue, but, in contrast to astrocytomas, they often have remarkably sharp borders with the surrounding brain tissue. ${ }^{7,11}$ ODs can arise, however, throughout the central nervous system including infratentorial sites and the spinal cord.

Most infratentorial ODs arise from the posterior brainstem with fourth ventricular extensions. Some differences are observed between supra- and infratentorial ODs. Posterior fossa tumors are more often cystic and less often calcified. ${ }^{12}$ Furthermore, posterior fossa ODs tend to be more aggressive. $^{13}$ Like supratentorial neoplasms, infratentorial neoplasms tend to infiltrate along white matter tracts, regardless of their histologic grades. ${ }^{14}$ Levidou et al reported a cystic low-grade OD with good demarcation of neurovascular structures including the tectal plate. ${ }^{5}$ The codeletion $1 \mathrm{p} 19 \mathrm{q}$ is associated with better tumor response to chemotherapy and more favorable prognosis. ${ }^{7,9}$

Resection of gliomas in the tectal plate can cause $50 \%$ of morbidity, such as Parinaud syndrome and other eye movement impairments, auditory hallucination, acoustic neglect syndrome and akinetic mutism, and 10\% mortality, as shown in some series. ${ }^{15}$ Therefore, it is recommended to avoid tumor resection beyond the inferior colliculi caudally, to prevent auditory damage, and ventrally to the aqueduct, to avoid damaging the third nerve nucleus. In the present case, the tumor has bilaterally infiltrated the central gray area, tectal plate, and pulvinar, probably following the fibers of the posterior commissure and pretectal area. These structures have very complex functions that are still not completely understood. The tectal region or quadrigeminal plate is dorsal to the aqueduct and marked rostrally by the posterior commissure, which contains fibers from pretectal nuclei, the nuclei of posterior commissure, the superior colliculi, and the interstitial nuclei of Cajal. Immediately rostral to the superior colliculi, in the area of the junction of the midbrain and the thalamus, is the pretectal area, which is involved in accommodation, pupillary reflexes, and vertical eye movement. The superior colliculi receives visual, auditory, and somatosensory stimuli related to head and eye movement toward the stimuli. These structures are related to the control of intrinsic and extrinsic musculature involved in eye reflexes. The cerebral aqueduct is surrounded by central gray matter. In its ventral part is the oculomotor nuclear complex, with its accessory oculomotor nuclei (Edinger-Westphal). The tumor's pattern of infiltration into these areas has prevented total tumor removal.

\section{Conclusions}

Although multiple studies have shown that the extent of resection of a glioma is important to the prognosis, ${ }^{16,17}$ resecting portions of a tumor that infiltrates functional areas 
like the tectal plate must be done judiciously. When there are no sharp boundaries between the tumor and the brainstem tissue, it is prudent to pursue only safe subtotal resection because most ODs respond well to both radiation and chemotherapy. Ongoing surveillance is mandatory in this situation because a recurrence is possible. ${ }^{6,7}$ In the current patient, although a subtotal resection was performed, she presented complete remission of the residual lesion after radiation and chemotherapy at 24-month follow-up.

\section{References}

1 Gaillard F, Jones J. Masses of the pineal region: clinical presentation and radiographic features. Postgrad Med J 2010;86(1020):597-607

2 Regis J, Bouillot P, Rouby-Volot F, Figarella-Branger D, Dufour H, Peragut JC. Pineal region tumors and the role of stereotactic biopsy: review of the mortality, morbidity, and diagnostic rates in 370 cases. Neurosurgery 1996;39(5):907-912; discussion 912-914

3 Sano K. Pineal Region and Posterior Third Ventricular Tumors: A Surgical Overview. Baltimore, MD: Williams \&Wilkins; 1987

4 Das S, Chandler JP, Pollack A, et al. Oligodendroglioma of the pineal region. Case report. J Neurosurg 2006;105(3):461-464

5 Levidou G, Korkolopoulou P, Agrogiannis G, Paidakakos N, Bouramas D, Patsouris E. Low-grade oligodendroglioma of the pineal gland: a case report and review of the literature. Diagn Pathol 2010;5:59

6 Engelhard HH, Stelea A, Cochran EJ. Oligodendroglioma: pathology and molecular biology. Surg Neurol 2002;58(2):111-117; discussion 117
7 Baehring JM. An update on oligodendroglial neoplasms. Curr Opin Neurol 2005;18(6):639-644

8 Chin HW, Hazel JJ, Kim TH, Webster JH. Oligodendrogliomas. I. A clinical study of cerebral oligodendrogliomas. Cancer 1980;45(6): $1458-1466$

9 Gan HK, Rosenthal MA, Dowling A, et al. A phase II trial of primary temozolomide in patients with grade III oligodendroglial brain tumors. Neuro-oncol 2010;12(5):500-507

10 Reis Filho JS, Netto MR, Sluminsky BG, et al. Oligodendroglioma: a pathological and clinical study of 15 cases [in Portuguese]. Arq Neuropsiquiatr 1999;57(2A):249-254

11 Van den Bent MJ, Reni M, Gatta G, Vecht C. Oligodendroglioma. Crit Rev Oncol Hematol 2008;66(3):262-272

12 Krueger EG, Krupp G. Oligodendrogliomas arising from structures of the posterior fossa. Neurology 1952;2(6):461-470

13 Packer RJ, Sutton LN, Rorke LB, et al. Oligodendroglioma of the posterior fossa in childhood. Cancer 1985;56(1):195-199

14 Alvarez JA, Cohen ML, Hlavin ML. Primary intrinsic brainstem oligodendroglioma in an adult. Case report and review of the literature. J Neurosurg 1996;85(6):1165-1169

15 Lapras C, Bognar L, Turjman F, et al. Tectal plate gliomas. Part I: Microsurgery of the tectal plate gliomas. Acta Neurochir (Wien) 1994;126(2-4):76-83

16 Berger MS, Deliganis AV, Dobbins J, Keles GE. The effect of extent of resection on recurrence in patients with low grade cerebral hemisphere gliomas. Cancer 1994;74(6):1784-1791

17 Kumar P, Tatke M, Sharma A, Singh D. Histological analysis of lesions of the pineal region: A retrospective study of 12 years. Pathology - Research and Practice 2006;202:85-92 